\title{
Upskilling nursing students and nurse practitioners to initiate and manage patients on ART: An outcome evaluation of the UKZN NIMART course
}

R Mngqibisa, ${ }^{1} \mathrm{MB} \mathrm{ChB}, \mathrm{MPH} ;$ M Muzigaba, ${ }^{1} \mathrm{PhD}, \mathrm{MPH}$, MPhil, BSc; B P Ncama, ${ }^{2}$ BCur, MCur, MBA, PhD; S Pillay, ${ }^{1}$ MB ChB, DOM, Dip HIV Man; N Nadesan-Reddy, ${ }^{1} \mathrm{MB}$ ChB, FCPHM, MMed (Public Health Med)

${ }^{1}$ Medical Education Partnership Initiative, School of Clinical Medicine, College of Health Sciences, University of KwaZulu-Natal, Durban, South Africa ${ }^{2}$ School of Nursing and Public Health, College of Health Sciences, University of KwaZulu-Natal, Durban, South Africa

Corresponding author: R Mngqibisa (rmngqibisa@gmail.com)

\begin{abstract}
Background. Currently, there is a need in South Africa to implement strategies to upskill nurses in the clinical management of HIV and AIDS, for effective and efficient management of people living with HIV. One such strategy is the nurse-initiated management of antiretroviral therapy (NIMART) course. Objectives. To evaluate the effectiveness of the NIMART course in increasing the knowledge of trainees in select clinical competencies, to assess whether perceived knowledge gain varied according to individual-level characteristics of trainees, and to determine trainee perceptions of the value and delivery of the course.

Methods. A 5-day training course focusing on various areas of HIV was developed and delivered by experts in the field of HIV to multiple cohorts of fourth-year nursing students at the University of KwaZulu-Natal, and to post-basic nursing practitioners, over a 5-year period. A single-group pre- and post-quasi-experimental design was used to assess knowledge change and perceptions about the course among 1369 trainees who had benefitted from the course during the implementation period.

Results. Post-workshop test scores were significantly higher than pre-workshop scores $(p<0.0001)$, based on both pooled and cohort-specific data. For pooled analysis, the pre-test median score was $67 \%$ (interquartile range (IQR) $=60 \%-73 \%$ ) and the post-test median score was $77 \%(I Q R=70 \%-80 \%)$, with $p<0.0001$. The knowledge gain was the highest in respect of HIV prevention, followed by prevention of mother-to-child transmission, then HIV treatment and lastly, general knowledge of HIV. The vast majority were very satisfied with the content of the training, although $31.3 \%$ strongly disagreed that they were ready to apply the knowledge they had learned in their workplace.

Conclusion. The training was generally well received, and improved the knowledge of participants in HIV and its management. However, this outcome represents short-term benefits of the programme, and there is a need for on-the-job mentorship and support in order to maximise on clinical outcomes related to HIV.
\end{abstract}

Afr J Health Professions Educ 2017;9(3):153-158. DOI:10.7196/AJHPE.2017.v9i3.879

HIV/AIDS continues to be a global public-health problem. Globally, there are 36.7 million people living with HIV, 25.8 million of whom live in subSaharan Africa (SSA). ${ }^{[1]}$ As of June 2015, 17 million people living with HIV were accessing antiretroviral therapy (ART), with 10.3 million of these accessing antiretroviral treatment in SSA. This is $54 \%$ of all people living with HIV in the region. ${ }^{[1]}$ HIV/AIDS is also responsible for a large percentage of morbidity and mortality, especially in settings that have limited resources. ${ }^{[2]}$ Of further concern is the fact that SSA was also home to $70 \%$ of all new HIV infections in 2012. ${ }^{[3]}$

Compared with other countries in the world, South Africa (SA) has the highest number of people living with HIV and AIDS, and currently, the country has the largest public health antiretroviral therapy (ART) programme, which began in $2004^{[4,5]}$ and is being received by about 3.4 million people. $^{[1]}$

With an estimated 6.4 million people living with HIV (PLHIV) in SA by mid-2012, ${ }^{[6]}$ and increasing numbers of people requiring ART, treatment services need to be decentralised to ensure access and expansion of ART services. Barriers for the majority of South Africans needing healthcare, such as poverty and the inability to travel long distances to access healthcare facilities, add to the critical need to decentralise HIV and AIDS care to primary healthcare settings. ${ }^{[7]}$ Additionally, with the recent changes in eligibility criteria for ART initiation, and the resultant increase in the number of patients requiring antiretroviral (ARV) medication, a large pool of trained nurses will be required to initiate and manage patients requiring ART. In a recent media release published by the KwaZulu-Natal Department of Health (KZN DoH), the Provincial Health Member of the Executive Committee in KZN, Minister Dr Sibongiseni Dhlomo, announced that 'the KZN DoH must urgently train many more nurses in the administering of antiretroviral treatment as SA adopts the World Health Organization's progressive "test and treat" HIV guidelines. ${ }^{\text {'[8] }}$ According to the same report, at the beginning of 2016, more than 1700 nurses had been trained in nurseinitiated management of ART (NIMART) - a task-shifting strategy put in place in 2010 by the SA government to enhance care for PLHIV - but there remained a need to double this number by the end of 2016. ${ }^{[8]}$

The NIMART strategy involves nurses initiating patients on ART, providing repeat prescriptions for patients stable on ART and appropriate 
referral to physicians as required. ${ }^{[9]}$ Although there were still concerns about the ability of nurses to prescribe ART, some studies have reported equivalent treatment outcomes for patients treated by nurses to those managed by doctors. ${ }^{[10-13]}$ Studies in SA have also reported similar outcomes, although some of these findings do not represent standard practice, as they are from sites/clinics heavily supported by non-governmental organisations and where intensive training and ongoing support of nurses was implemented. ${ }^{[11-14]}$

A study from Cape Town showed that the responses of nurses to taskshifting varied from embracing it as a challenge capable of making their work more interesting, and increasing their knowledge and skills, to resisting it because of the additional workload without adequate support and training. ${ }^{[15]}$ Koome et al., ${ }^{[16]}$ in their study in Kenya, showed that the majority of nurses $(70 \%, n=162)$ understood and supported task-shifting. Support for task-shifting increased with increased years of experience, and was strongly correlated to knowledge ${ }^{[16]}$ while support, supervision and training were seen by study participants as requirements for effective task-shifting. ${ }^{[17]}$ Educational and occupational healthcare-worker training programmes have been shown to decrease fear and improve knowledge regarding HIV and AIDS transmission, and have led to the overcoming of negative attitudes and increased confidence and ability to care for PLHIV. ${ }^{[16]}$

This article outlines the University of KwaZulu-Natal's (UKZN's) NIMART training model, designed to contribute towards the task-shifting and task-sharing strategy for better HIV treatment outcomes in SA. The article also presents results from a summative evaluation of the NIMART course, with a particular focus on the number and background of trainees who benefitted from the course from 2011 to 2015, and an assessment of the extent to which the course has improved the knowledge of trainees in the management of HIV-positive people. Trainee characteristics associated with knowledge change, and their perceptions about the delivery of the course, are also presented in this report.

The NIMART course at UKZN was led by the Enhancing Care Initiative, and supported by a Medical Education Partnership Initiative (MEPI) grant. The course was launched at UKZN in 2011, and between 2011 and 2015, at least three separate courses were delivered each year. Each course lasted 5 days, and targeted both the fourth-year undergraduate nursing students and registered nurses who were already practising and doing post-basic and postgraduate programmes. The course focused on a number of topics related to the management of ART, and was taught by experts in the field of HIV and AIDS. The content covered during the 5-day course was complementary to the HIV content integrated into the curriculum.

The course covered: (i) the basic science of HIV infection, including epidemiology, immunology and virology, and clinical aspects of HIV infection and AIDS; (ii) HIV management in adults and children, which includes opportunistic infections and their presentation and management; (iii) ART in adults and children, which includes classes of drugs and their modes of action; (iv) latest SA guidelines; ( $v$ ) drug-related toxicities and their management, including treatment failure; (vi) HIV in women; (vii) HIV and TB comorbidity; (viii) ethics related to HIV and AIDS; and (ix) palliative care for PLHIV

\section{Methods Setting}

This evaluation involved data collected at UKZN from NIMART participants who benefitted from the course during the period 2011 - 2015.

\section{Design}

A single-group pre- and post-test quasi-experimental design was used to evaluate the extent to which training participants from different cohorts gained knowledge related to HIV clinical management. This type of study design has been widely used in estimating the effect of interventions on short-term outcomes, such as knowledge and attitudes, despite its limitations in alleviating a number of internal-validity threats such as selection bias and subject regression to the mean. ${ }^{[18]}$ Change in knowledge was assessed by comparing pre- and post-test scores of NIMART trainees who received the training between 2012 and 2015. The data used for this analysis were therefore secondary data collected as part of outcome and process monitoring and scheduled evaluations of the NIMART course during this period. The assessment of trainee perceptions of the delivery and value of the NIMART course, as well as factors associated with perceived knowledge gain, involved cross-sectional analysis of pooled data collected from 2011 to 2015 .

\section{Ethical considerations}

The dataset used for this evaluation was collected for the purposes of quality assurance for the MEPI programme. The dataset was obtained and used in robustly anonymised form, with all the identifying information removed. As the assessment of knowledge gain required paired analysis, pre- and post-test scores for individual trainees were supplied without names or unique identifiers which could be linked to participants' details. A complete application for ethics approval was submitted to the UKZN Biomedical Research Ethics Committee, which granted ethics approval for this evaluation to be conducted (ref. no. BE035/15).

\section{Participants}

A non-probability sample that consisted of all 615 undergraduate nursing students, as well as 754 practising and post-basic nursing students, who benefitted from the NIMART course during the 2011 - 2015 course rollout period, was used. In total, 1369 NIMART trainees were involved in this evaluation. All participants whose anonymised data were available in the programme monitoring records spanning the evaluation period (2011 2015) were considered for this evaluation.

\section{Data collection}

The data were collected from successive cohorts of learners who attended the NIMART course between 2011 and 2015. Three data-collection tools were used to collect these data. The first tool was a structured participantregistration form, which was used to collect information on the demographic and professional characteristics of NIMART trainees. The second tool was an anonymous knowledge-assessment questionnaire, which was completed by trainees before and after the course. This tool consisted of 30 'true or false'- and 'don't know'-type questions that represented key competencies for NIMART, including general knowledge of HIV/AIDS, knowledge of HIV treatment, HIV prevention and prevention of mother-to-child transmission (PMTCT) of HIV, to mention but a few. This tool was piloted in a sample of 15 participants, and evaluated for internal consistency using Cronbach's alpha. Both positively and negatively worded questions were used, and the tool was given to all participants in each cohort of trainees to quantify their change in knowledge following the course. The third tool was a course-evaluation questionnaire, which collected information 
on participants' perceptions of the value and delivery of the course, their readiness for NIMART, their perceived knowledge change following the course and their satisfaction with the content of different NIMART thematic areas of training. This tool was also assessed for internal consistency using the Cronbach alpha test. The same three research instruments were used over the course of the evaluation period to ensure that measurements across cohorts of learners were standardised.

\section{Data analysis}

All the data were firstly processed in Excel 2010 (Microsoft, USA) and exported to Stata/IC 13.0 (StataCorp LLC, USA) and Tableau version 9.3 (Tableau Software, USA) for analysis. Trainee demographic and professional characteristics were summarised using frequency tables. The pre- and post-test scores and the score for perceived knowledge change were first inspected for normality using the Shapiro-Wilk and Shapiro-Francia tests. The distributions of the scores for perceived knowledge change across trainee demographic and processional characteristics were displayed using forest plots, with mean scores per group and 95\% confidence intervals.

The pre- and post-test scores were not normally distributed, and therefore the change in knowledge was assessed using the Wilcoxon signed-rank test for matched pairs. The distribution of scores before and after the training was displayed per cohort using a box plot with quantile plot overlays. This plot was chosen so as to show side-by-side quantile plots of cumulative distribution of pre- and post-test scores and their median, as well as the 25th and 75th percentiles. Trainees' satisfaction with the content of different NIMART thematic areas of training, as well as their perceptions of the value and delivery of the training, were summarised using a divergent bar graph. A $p$-value $<0.05$ was considered statistically significant.

\section{Results}

The Cronbach alpha tests carried out to assess the internal consistency of the study instrument revealed that the scales used in the instrument were reliable. The alpha coefficients for different sets of questions ranged between 0.78 and 0.86 .

A descriptive analysis of trainee characteristics revealed that by the year 2015, a total of 1369 trainees from across the KZN province had benefitted from the programme, $87 \%$ of whom were female and $61 \%$ were working in healthcare facilities in rural KZN. The course uptake was highest among registered nurses (76.05\%), compared with student nurses (12.37\%), clinical nurse practitioners $(8.68 \%)$, professional nurses $(0.79 \%)$ and other nursing categories $(2.11 \%)$.

\section{Assessment of perceived knowledge change}

From the mean percent scores presented in Fig. 1, it can be seen that Indian and white NIMART trainees believed that they gained relatively less knowledge than their coloured and black counterparts $(p=0.084)$. However, these differences were not statistically significant. Candidates who came from facilities based in urban and peri-urban areas also believed that they gained relatively less knowledge from the training than their counterparts from rural areas $(p=0.045)$, and male candidates believed that they learned more than females $(p=0.726)$, as did professional nurses compared with student nurses $(p=0.025)$. Similarly, participants who were in the last cohort believed they learned a lot more from the training compared with their colleagues in the preceding years $(p=0.002)$. The mean score $(89 \%)$ was relatively high, which indicates that, on average, trainees believed they learned something from the training.

\section{Assessment of actual knowledge change}

Fig. 2 illustrates overall knowledge change among participants, across a number of competencies related to NIMART. Based on the median scores, it can be seen that the knowledge increase was consistent and statistically significant across all four training periods $(p<0.0001)$. The median pre- and post-test scores were the highest in year 4 of the training programme.

Fig. 3 depicts the changes in knowledge in four broad areas of the training course. The knowledge gain was the highest in respect of HIV prevention, followed by PMTCT, then HIV treatment, and lastly, general knowledge of HIV.

\section{Perceptions of the course}

Participants' perceptions of the course were assessed based on 25 questions that represented different thematic learning areas of the course. The results from this assessment are presented in Fig. 4.

\begin{tabular}{|c|c|c|c|c|}
\hline Subgroup & $n$ & & Effect $(95 \% \mathrm{Cl})$ & $p$-value \\
\hline \multicolumn{5}{|l|}{ Race } \\
\hline $\begin{array}{l}\text { Black } \\
\text { Coloured }\end{array}$ & - & & $\begin{array}{l}89.08(88.17,89.98) \\
93.1688 .90,97.41\end{array}$ & \\
\hline $\begin{array}{l}\text { Coloured } \\
\text { Indian }\end{array}$ & 二— & & $\begin{array}{l}93.16 \text { (88.90, 97.41) } \\
85.65(81.91 .89 .39)\end{array}$ & \\
\hline Other & 10 & & $93.00(87.90,98.10)$ & \\
\hline White & $14 \leftarrow$ & - & $86.43(77.33,95.53)$ & 0.084 \\
\hline \multicolumn{5}{|l|}{ Cohort } \\
\hline 2012 & & & $81.25(67.67,94.83)$ & \\
\hline 2013 & $-\infty+$ & & $88.02(86.44,89.60)$ & \\
\hline 2014 & $-\infty-$ & & $\begin{array}{l}86.31(84.90,87.73) \\
9320\end{array}$ & \\
\hline 2015 & 283 & $-\infty-$ & $93.20(91.92,94.48)$ & 0.003 \\
\hline \multicolumn{5}{|l|}{ Gender } \\
\hline Female & 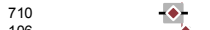 & & $88.44(87.49,89.38)$ & \\
\hline Male & 106 & & $89.25(86.78,91.71)$ & 0.726 \\
\hline \multicolumn{5}{|l|}{ Geographical area where employed } \\
\hline $\begin{array}{l}\text { Urban } \\
\text { Rural }\end{array}$ & $-\infty-$ & & $88.34(86.95,89.73)$ & \\
\hline Peri-urban & $\longrightarrow$ & & $85.80(82.60,89.01)$ & 0.045 \\
\hline \multicolumn{5}{|l|}{ Profession } \\
\hline $\begin{array}{l}\text { Registered professional nurses } \\
\text { Student nurses }\end{array}$ & ${ }_{48}^{195} \longleftarrow$ & & $\begin{array}{l}87.59(85.83,89.35) \\
83.33(78.65,88.01)\end{array}$ & 0.025 \\
\hline \multirow[t]{3}{*}{ Overall } & $\diamond$ & & $89.01(88.16,89.86)$ & \\
\hline & $\begin{array}{l}1 \\
80\end{array}$ & 95 & 100 & \\
\hline & \multicolumn{4}{|c|}{ Mean score for perceived knowledge gain (\%) } \\
\hline
\end{tabular}

Fig. 1. A forest plot showing trainee perceived knowledge change in the management of ART following the training.



Fig. 2. Strip plot showing the cumulative distribution of test scores and median test scores before and after the training with significant tests: pooled data by year of training. 
Fig. 4. shows that, based on the pooled data, the vast majority of trainees who received NIMART training were very satisfied, or just satisfied, with the content of the training across all the 25 thematic areas of learning that they were asked to evaluate.

A shown in Fig. 5, 31.3\% of the NIMART trainees strongly disagreed that they would be able to apply the knowledge and skills that they had learned from the training to their job upon returning to their workplace. Furthermore, 31\% of trainees were not sure whether the use of case studies during their training was useful to their learning. However, the vast majority believed that the handouts given would be useful to them, and that the use of the examples during the training contributed to their understanding of the training content.



Fig. 3. Knowledge gain in specific thematic areas of learning related to nurse-initiated management of antiretroviral therapy competencies, pooled analysis (2012 - 2015). The pooled analysis, which combined data from all 4 years of assessment without considering different thematic areas of learning, also showed that knowledge increased significantly, with a baseline median score of $67 \%$, which increased to $77 \%$ after the training $(\mathrm{p}<0.0001)$. The circles demarcate mean scores. PMTCT = prevention of mother-to-child transmission.

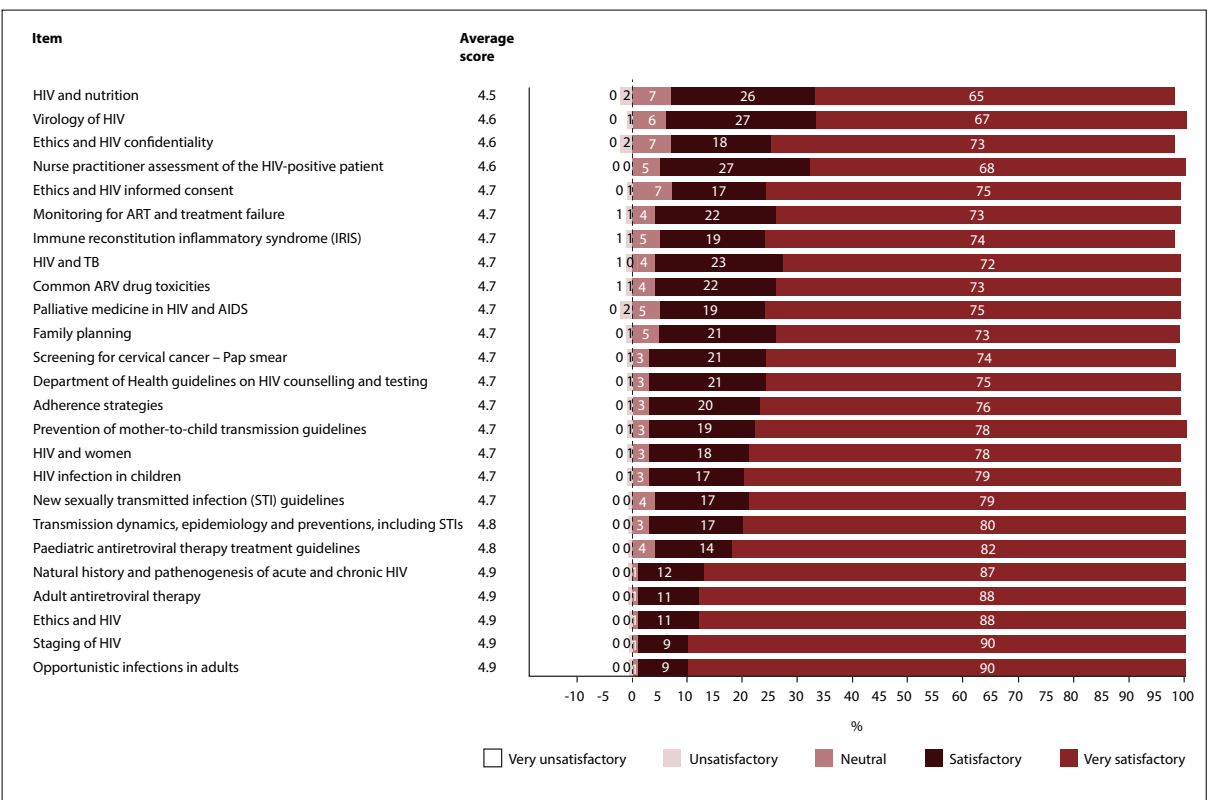

Fig. 4. Divergent bar graph showing trainees' satisfaction with the content of different thematic training areas of the nurse-initiated management of antiretroviral therapy course: pooled analysis (2011 - 2015). Each thematic area of learning was measured on a 5-point Likert scale with values 1 - 5.

\section{Discussion \\ Key findings}

This evaluation indicated an overall improvement of knowledge regarding all aspects of HIV management among nursing students and postbasic and working nurses who attended the NIMART training between 2012 and 2015 .

When looking at specific thematic areas of learning, the pre- and post-knowledge scores were highest for PMTCT and HIV prevention, which might be due to the fact that nurses have been expected to demonstrate competencies around PMTCT and HIV prevention since the beginning of the ART programme in SA..$^{[18,19]}$ Therefore, their knowledge in these two thematic areas would be expected to be relatively high. The scores were lower with respect to general HIV knowledge and HIV treatment, which contrasts with the findings from a study conducted in India that showed high baseline and follow-up knowledge of HIV and its treatment. ${ }^{[20]}$

Pooled results revealed relatively poor baseline knowledge $($ mean $=67)$. This may be related to inadequate learning among some trainees during their formative years, and limited exposure to the management of HIV-positive patients. Although not assessed in this evaluation - because the data were not available in the dataset used - it is hypothesised that baseline scores on objective measures of knowledge would have varied by professional characteristics and years of experience in the field. However, the evaluation focused on differences in knowledge according to when trainees received the training, and it was shown that both the baseline and post-test knowledge levels were better in the last group that benefitted from the training. This could be ascribed to the fact that by 2015 , the majority of the nursing students were already benefitting from a curriculum which incorporated HIV, and the nurses were mostly from the facilities where NIMART was already being practised.

In addition to the objectively measured knowledge gain, trainees were also asked to indicate the extent to which the course had benefitted them in terms of knowledge shift. The question asked them to rate, on a scale of $1 \%-100 \%$, how much they believed that their knowledge about HIV and its management had been impacted as a result of the NIMART course. Based on this assessment, it was determined whether their perception of the magnitude of knowledge change depended on their professional and demographic characteristics. 




Fig. 5. Divergent bar graph showing trainees' perceived value of the nurse-initiated management of antiretroviral therapy training programme in their workplace: pooled analysis (2012 - 2015).

Trainees from facilities based in urban and periurban areas reported less knowledge gain than their counterparts based in rural areas. This may be a proxy for superiority of knowledge at baseline, and may possibly be related to the fact that trainees in urban and peri-urban areas have better exposure to the management of HIV and possibly mentoring. It is therefore to be expected that they are likely to report less knowledge gain from the training compared with those in rural areas, who most likely have fewer support mechanisms around them to promote learning. Thus it may be correct to assume that more NIMART training is offered in urban/peri-urban areas than in rural areas, while the need for information may be greatest in the latter.

\section{Recommendations}

Pooled analysis also revealed that the median post-training score was $77 \%$. For HIV treatment it was $75 \%$, and $78 \%$ for PMTCT. It may be argued that these scores are not sufficient within the context of effective treatment of PLHIV and may suggest that didactic training alone is not adequate to capacitate or improve the ability of nurses to manage HIV. Training needs to be supported with continuous and ongoing mentoring, especially since only $63 \%$ of nurses felt confident enough to apply the knowledge and skills gained to manage PLHIV post training. This argument is supported by a study in Limpopo Province, SA, which showed that better knowledge was associated with frequency of training. ${ }^{[21]}$ Another study by Cameron et al..$^{[22]}$ also argues that expanding clinical mentoring and further training in clinical skills and pharmacology would assist in reaching the target of initiating more HIV-positive patients on ART. The baseline results on HIV clinical knowledge also highlight the need to create a strong foundation for nurses during their formative years, so that they are equipped with the requisite competencies to effectively manage PLHIV. The nursing curricula in the various training institutions that are producing the next generation of nurses need to be structured to ensure that there is sufficient coverage of the essential elements of management of HIV, coupled with ongoing in-service training of nurses in the management of HIV, such as the NIMART programme.

It is also recommended that a much more extensive study be carried out, to not only focus on the shift in knowledge related to HIV and its management, but also to track and trace NIMART trainees to establish whether they have implemented this knowledge in their workplaces, and to determine whether such an intervention has had an impact on an array of health outcomes. In SA, there is still a dearth of literature around the effectiveness of NIMART training in improving clinical outcomes. One study has, however, demonstrated that NIMART training can increase ART uptake and reduce workload at referral facilities, enabling doctors to concentrate on more complicated cases. ${ }^{[23]}$ In their survey conducted in SA to assess whether nurses in primary-care clinics were initiating ART after attending NIMART training, Cameron et al. ${ }^{[22]}$ showed that of the nurses surveyed, $62 \%(79 / 126)$ had started initiating new adult patients on ART, but only 7\% (9/126) were initiating ART in children. The main barrier to initiation was allocation to other tasks in the clinic as a result of staff shortages.

\section{Limitations}

One of the limitations of this evaluation relates to the generalisability of the study findings. This is primarily because the study used a convenient sample of nurse practitioners and post-basic nurses who attended the NIMART training course at UKZN during a specific period of time. However, the study does account for all the nursing students who were trained at UKZN during the evaluation period. Another limitation is that the study did not assess whether there were any differences between the nursing students and post-basic nurses and practising nurses in respect of baseline knowledge gain as well as actual knowledge shift. It would also have been valuable to show whether knowledge differed among practising nurses according to their level of experience in the clinical setting. This evaluation used secondary data, and these variables were not captured as part of primary data collection, and therefore could not be used in this study.

\section{Conclusion}

The UKZN NIMART training course has played a significant role in capacitating more than 1300 nurses in KZN Province with NIMART skills, against the provincial target to double the number of NIMART-trained nurses from 1600 by the end of 2016. This evaluation showed that the NIMART training programme can significantly increase knowledge of HIV and its clinical management among nursing students and nursing practitioners pursuing their post-basic training in the nursing field. The study showed that their baseline knowledge was low, and that perceptions about learning varied among trainees depending on their demographic and professional characteristics. Based on participants' perceptions of how much knowledge shift they experienced from the course, it was evident that nurses from rural areas had the greatest need for the course. Despite the observed increase in knowledge, however, a certain proportion of trainees did not feel ready to implement the knowledge gained in their workplace, which suggests a need for an 'on-the- 
job' mentorship programme if the benefits of the programme are to be carried forward and be translated into positive health outcomes.

Acknowledgements. This work was made possible by grant no. 5R24TW008863 from the President's Emergency Plan for AIDS Relief (PEPFAR) and the National Institutes of Health, US Department of Health and Human Services. Its contents are solely the responsibility of the UKZN MEPI programme, and do not necessarily represent the official views of the government. The KZN Department of Health is also acknowledged for making the NIMART programme successful. Author contributions. RM, BN, SP and NN were involved in the development and roll out of the UKZN-NIMART programme, RM and BN led the implementation of the programme, MM and RM conceived the evaluation, MM conducted the study, and analysed the data, and MM, RM and BN drafted the manuscript for all co-authors to edit and sign off.

Funding. This work was made possible by grant no. 5R24TW008863 from PEPFAR and the National Institutes of Health, US Department of Health and Human Services.

Conflicts of interest. The authors declare no conflict of interest related to this work.

1. United Nations Programme on HIV/AIDS. Global AIDS update. Geneva: UN, 2016. http://www.unaids.org sites/default/files/media_asset/global-AIDS-update-2016_en.pdf (accessed 12 July 2016).

. World Health Organization. Millennium Development Goals (MDGs). Fact sheet No. 290. New York: Unite Nations, 2015. http://www.who.int/mediacentre/factsheets/fs290/en/ (accessed 14 August 2016).

. United Nations Programme on HIV/AIDS. Joint United Nations Programme on HIV/AIDS: UNAIDS Partnership with the Global Coalition on Women and AIDS (GCWA). Geneva: UNAIDS, 2010. http://www womenandaids.net/About-GCWA/Partners-and-Members/Joint-United-Nations-Programme-on-HIV-AIDS\%28UNAIDS.aspx (accessed 14 August 2016).

4. Health Systems Trust. Public HAART Projects in South Africa - progress to November 2004. Durban Health Systems Trust, 2005. http://www.hst.org.za/publications/public-haart-projects-south-africa-progress-

5. Uebel KE, Timmerman V, Ingle SM, J van Rensburg DHC, Mollentze WF. Towards universal ARV access: Achievements and challenges in Free State Province, South Africa. S Afr Med J 2010;100(9):589-593. https:// doi.org/10.7196/samj.3897

Pretoria: South Africa National AIDS Council, 2011. https://www.health-e.org.za/wp-content/uploads/2014/12/SANAC-NSP-ProgressReport-2014.pdf (accessed 2 September 2016).
7. Doshi RK, del Rio C, Marconi VC. Social determinants of HIV healthcare: A tale of two cities. In: Kasenga FY, ed. Understanding HIV/AIDS Management and Care - Pandemic Approaches in the 21st Century. Atlanta: Emory University School of Medicine and Rollins School of Public Health, 2011:34-58.

8. KwaZulu-Natal Department of Health. More nurses to be trained on nurse-initiated management of antiretroviral treatment (NIMART) programme to meet the expected high demand, following the adoption of new HIV 'Test and Treat' guidelines. Media release: KZN DoH. Pietermaritzburg: KZN DoH, 2016. http://www kznhealth gov za/mediarelease/2016/NIMART-test-treat-guidelines-17052016.htm (accessed I December 2016).

9. Zuma J. Address by President Jacob Zuma on the occasion of World Aids Day, Pretoria Showgrounds, 1 December 2009. http://www.info.gov.za/speeches/2009/09120112151001.htm (accessed 17 March 2016).

10. Georgeu D, Colvin CJ, Lewin S, et al. Implementing nurse-initiated and managed antiretroviral treatment (NIMART) in South Africa: A qualitative process evaluation of the STRETCH trial. Implement Sci 2012; 7(66). https//doi.org/10.1186/1748-5908-7-66

11. Callaghan M, Ford N, Schneider H. A systematic review of task-shifting for HIV treatment and care in Africa. Hum Resour Health 2010;8(8):1-9. https://doi.org/10.1186/1478-4491-8-8

12. Shumbusho F, van Griensven J, Lowrance D, et al. Task shifting for scale-up of HIV care: Evaluation of nursecentered antiretroviral treatment at rural health centers in Rwanda. PLoS Med 2009;6(10):e1000163. https://doi org/10.1371/journal.pmed.1000163

13. Cohen R, Lynch S, Bygrave H, et al. Antiretroviral treatment outcomes from a nurse-driven, community supported HIV/AIDS treatment program in rural Lesotho: Observational cohort assessment at two years. J Int AIDS Soc 2009;12(1):23. https://doi.org/10.1186/1758-2652-12-23

14. Uebel KE, Lombard C, Joubert G, et al. Integration of HIV care into primary care in South Africa: Effect on survival of patients needing antiretroviral treatment. J Acquir Immune Defic Syndr 2013;63(3):94100. https:/ doi.org/10.1097/qai.0b013e318291cd08

15. Colvin CJ, Fairall L, Lewin S, et al. Expanding access to ART in South Africa: The role of nurse initiated treatment S Afr Med J 2010;100(4):210-212. https://doi.org/10.7196/samj.4124

16. Centers for Disease Control. Recommended Prevention Services. Atlanta: Centers for Disease Control and Prevention, 2013. http://www.cdc.gov/hiv/prevention/programs/pwp/risk.html (accessed 10 August 2016).
.

17. Koome DI. Feasibility of task shifting in nursing practice: A case study of two level five public hospitals in Kenya. Kenya: University of Nairobi, 2012. http://erepository.uonbiac.ke/handle/1 1295/9328 (accessed 10 August 2016).

18. Shadish RW, Cook DT, Campbell DT. Experimental and Quasi Experimental Designs for Generalized Causal Inference. Boston: Houghton Mifflin Company, 2002.

19. Phetlhu DR, Watson MJ. Perceptions and attitudes of health workers towards patients co-infected with HIV and tuberculosis. J Soc Sci 2011;29(1):23-7.

20. Dicicco-Bloom B, Crabtree B. The qualitative research interview. Med Educ 2006;40(4):314-321.

21. Mulaudzi MV, Pengpid S, Peltzer K. Nurses' knowledge, attitudes, and coping related to HIV and AIDS in a rural hospital in South Africa. Ethno Med 2011;5(1):25-32.

22. Cameron D. Nurse initiation and maintenance of patients on antiretroviral therapy: Are nurses in primary care clinics initiating ART after attending NIMART training? S Afr Med J 2012;102(2):98-100. https://doi. org/10.7196/samj.5195

23. Nyasulu JCY, Muchiri E, Mazwi S, Ratshefola M. NIMART rollout to primary healthcare facilities increases access to antiretrovirals in Johannesburg: An interrupted time series analysis. S Afr Med J 2013;103(4):232-236. https://doi.org/10.7196/SAMI.6380

Accepted 15 November 2016 